\title{
Collaborative robotics in wire harnesses spot taping process.
}

\author{
V.Román Ibáñez ${ }^{\mathrm{a}, *}$, F.A. Pujol ${ }^{\mathrm{b}}$, S.García Ortega ${ }^{\mathrm{a},}$, J.M.Sanz Perpiñán ${ }^{\mathrm{a},}$ \\ ${ }^{a}$ Technological Centre of Furniture and Wood, Yecla, Spain \\ ${ }^{b}$ Department of Computer Technology, University of Alicante, Alicante, Spain
}

\begin{abstract}
Wires harnesses are used in several industrial sectors such as automotive, white goods, toys, or electrical and mechanical engineering. One of the key tasks of the process is to assemble and secure harnesses with a taping pistol in several spots to group single wires and make the final harness with all the required ramifications. The proposed method tries to advance the state of the art and enhance the current process, which is being performed manually, by adding robotic arms in a custom cell that collaborates with the human worker. The robotic solution of the proposal will perform all the spot tapings of the process, while the worker performs only the positioning of the cable. It reduces the processing time of the task and allows workers to work on two harnesses at the same time. The results of the present proposal will have a positive impact on companies dedicated to the production of wiring and the sectors to which they supply their products. The solution will also impact on the ergonomic conditions of workers through an innovative work environment that removes the most tedious and repetitive tasks of the operator.
\end{abstract}

Key words: Collaborative robots, Manufacturing, Ergonomics, Wire harness

\section{Introduction}

Robots still find challenging to work with flexible materials because the unpredictability of the initial state in a real operation in terms of pose and shape, compared the conditions es5 tablished in the training process $[1,2]$. Among those flexible materials, automated electrical wire handling has been a subject of intensive research in the last few years, especially in the automotive sector.

After the revision of related works on this field, in [3] a

10 survey can be found on the production process of automotive wire harness.

Accordingly, Wire harness optimization of spot taping process has been achieved in [4] by using genetic algorithms to optimise the taping route and jigs layout [5]. However this ap15 proach did not include the way to calculate the maximum strain, which must be introduced manually. Other applications related ${ }_{45}$ to wire harnesses are the routing performed by artificial intelligence systems [6], the assembly of harnesses inside the body of cars [7], weight analysis and reduction of harnesses to re20 duce car payload [8], robotised recognition of wire harnesses [9], task guidance interfaces for assembly [10], wire harness design software [11] among others. Cycle time is one of the most important factors in wire harnesses production. Automotive sector takes this seriously by using optimization and line balancing with Kaizen approaches to improve it [12] which can be transferred to other sectors using wire harnesses. Correct ergonomics are a key component of manufacturing processes with workers involved, specially on repetitive tasks such as the

\footnotetext{
${ }^{*}$ Corresponding author
}

Email address: v.roman@cetem.es (V.Román Ibáñez) one described as it leads to negative effects on the health of workers both in the short and long term [13]. Several methods are available to objectively measure the ergonomics of a task or process $[14,15]$ whose score is useful to compare different approaches and test enhancements before implementing the one with better ergonomics. Those methods have been used for risk assessment on different real world scenarios [16-20].

One of the technologies that helps with both enhancing cycle time and ergonomics is collaborative robotics [21]. This technology is being used in Industry 4.0[22] to improve productivity in sectors with high customization and to enhance ergonomics by removing from the equation the part of the work that is repetitive and does not add value to the final product [23]. No collaborative solutions have been found in the state of the art for the specific problem. The Poka-Yoke method [24] is used in multiple industrial applications of the sector to improve the quality tool of operations in the process by reducing worker mistakes while handling materials during the task process. Using robotics to reduce the number of operations to be performed by the operator will minimise the need to use these type of methods as well as improve the quality of the final product.

Low cost embedded systems such as Raspberry Pi have been used in industrial robotic automation solutions [25-27] allowing to run complex industrial systems with modest computing boards and reducing the overall cost of the robotic solution, which is a key point when implementing on SMEs.

Additive manufacturing [28] helps in the prototyping part of process automation, specially due to cost restrictions and in scenarios where iterative and incremental methodologies are used during the process [29]. 
sues before the manufacture process takes place in a real robotic cell. This way, cost production may be reduced whereas the quality of the final product is increased.

This article is organised as follows: Section 2 gives a de65 tailed explanation of the design phase of software, hardware and additive manufacturing parts of the proposed solution. Section 3 measures and compares the cycle time of the actual manual process and the proposed method. In Section 4, a comparative of both methods is performed from the ergonomics point of 70 view. Section 5 showcases different alternative setups to scale the solution and adapt to other scenarios, and conclusions are drawn in Section 6.

\section{Solution design}

The proposed solution has been designed to allow operators to work with two wire harnesses at the same time. This is intended to improve the cycle time of the process, reducing costs and increasing benefits. By using collaborative robotics the process is split in two separate tasks with the former being the handling and positioning of the wire harnesses on the panels to work with and the latter, the spot taping process. This way the human operator is performing the part of the job that adds value to the task, as handling of ductile materials such as wires is very difficult to automate, while the collaborative robotic arm is performing the repetitive task that does not require of human skills. This design improves the ergonomics of the process for the human worker, since it removes part of the manual process and therefore minimises the movements required by the human operator. Additional improvements in ergonomics are bound to the design by adjusting the required pose

90 of the worker to handle the robotic cell during the process. The design of the proposed solution is described in detail in the next subsections, which include all the information about software modules, robotic cell hardware components and additive manufacturing used for the prototype.

\subsection{Software modules}

The robotic cell prototype is commanded by three separated software modules. The main module is the cell control software which is the one that is in direct contact with all the hardware parts of the cell as shown in Fig.1. It gets signals from the pedal that tells the software that the operator has finished his part of the process and wants to move the panel to one side so a cobot starts the spot taping process while the operator receives the batch to handle. The two panels are joined together and their movement is handled by a linear axis. This axis is controlled by the cell control software which moves it to two pre-recorded work positions and constantly checks if the axis has finished its movement to continue with the process. The controller is also managing the movement of the two collaborative robots, sending the desired path for the spot-taping process when necessary and checking whether it has finished or not to continue with the process. A FSM (Finite State Machine) [31] has been used as shown in Fig. 2 to ensure the correct behaviour of the robotic cell elements with the expected transitions between states. This



Figure 1: Software modules of the prototype and their relationships.



Figure 2: FSM diagram of the controller states and transitions.

FSM ensures that the cell does not move the linear axis when the pedal is pushed while it is already moving as well as when a robotic arm is still working even if the human operator pressed the pedal to continue with the process, avoiding dangerous situations for the operator and the cell elements [32]. The robot control software is responsible for receiving the trajectory from the cell control software, moving the robots and notifying the controller software that the process has finished. The last software module lets users configure parameters of the robotic cell through its graphical user interface such as the size and tilt of panels, spot time delay and offsets to adjust the virtual simulation to the physical elements of the cell, since slight differences might appear due to imprecision in the materials or in the assembly process. This software module allows users to change the layout of the spots used to perform the taping process in shape, position, orientation and order as can be seen in Fig.3. It allows cell to adapt to different wire harnesses models and configurations without having to reprogram the process, saving time and money. Software generates the trajectory that will be used to move the cobots and perform the spot-taping process, allowing to visualise and simulate in a $3 \mathrm{D}$ environment. The generated trajectory can be sent to the robotic cell controller 




Figure 3: GUI for spot-taping layout configuration.

software to be stored for later use in the operational process. For each spot of the bidimensional layout designed inside the cell configuration software, two points should be calculated to project position and rotation from $2 \mathrm{D}$ space to $3 \mathrm{D}$ space to generate the trajectory used to move both cobots.

$$
p_{\text {in }}=\left[\begin{array}{ccc}
1 & 0 & 0 \\
0 & \cos \left(\theta_{\text {tilt }}\right) & -\sin \left(\theta_{\text {titl }}\right) \\
0 & \sin \left(\theta_{\text {tilt }}\right) & \cos \left(\theta_{\text {tit }}\right)
\end{array}\right]\left[\begin{array}{ccc}
\cos \left(\theta_{\text {spot }}\right) & -\sin \left(\theta_{\text {spot }}\right) & 0 \\
\sin \left(\theta_{\text {spot }}\right) & \cos \left(\theta_{\text {spot }}\right) & 0 \\
0 & 0 & 1
\end{array}\right]\left[\begin{array}{c}
P_{x} \\
P_{y} \\
0
\end{array}\right]
$$

Equation Eq. 1 is used to obtain a $3 \mathrm{D}$ vector $p_{\text {in }}$ describing the projected 2D spot position $P$ and its rotation $\theta_{\text {spot }}$ after applying the tilt angle $\theta_{\text {tilt }}$ of the jig used for positioning and spot taping wire harnesses on the robotic cell.

$$
p_{\text {out }}=p_{\text {in }}-\left[\begin{array}{ccc}
1 & 0 & 0 \\
0 & \cos \left(\theta_{\text {till }}\right) & -\sin \left(\theta_{\text {ill }}\right) \\
0 & \sin \left(\theta_{\text {tilt }}\right) & \cos \left(\theta_{\text {tilt }}\right)
\end{array}\right]\left[\begin{array}{l}
0 \\
0 \\
1
\end{array}\right] t_{\text {offset }}
$$

In equation Eq. 2 a second point $p_{\text {out }}$ is obtained for each spot to slide along the $Z$ direction vector transformed with the $\theta_{\text {tilt }}$ of the jig an amount of millimetres determined by the taping offset $t_{\text {off set }}$ to separate the taping pistol from the jig in the direction that the taping pistol should approach to the jig. For each point in the generated trajectory, the cobot will move the taping pistol to $p_{\text {out }}$ and then, it will move to $p_{\text {in }}$ by using lin- ${ }_{125}$ ear interpolation to get close to the jig and ending with a final movement back to $p_{\text {out }}$ to move out of the jig and get ready to repeat the process with the remaining points. Figure 4 shows a generated trajectory by using this method inside a 3D viewport using a custom 3D engine based on OpenGL [33] included in the software package to preview the resulting trajectories before sending them to cobots.

\subsection{Robotic cell hardware components}

This section showcases the design and construction process of all the hardware parts of the prototype created to experiment and test the proposed solution. The main design of the cell is shown in Fig.5. It shows a working environment made of ${ }_{135}$ aluminium profiles with several components attached to it. A linear axis is attached to this chassis with 1 meter of horizontal movement and a speed of $500 \mathrm{~mm} / \mathrm{s}$. In top of the axis, a pair of aluminium panels rest, both moving in the same direction and alternating sides on each process step. The robotic cell has $\mathrm{a}_{140}$



Figure 4: 3D visualization of the dynamically generated trajectory.



Figure 5: Design of hardware parts of proposed robotic cell.

operator to tell the controller that the current step is finished and to move to the next part of the process. Cell is using two collaborative robots, one at each side, because it is required to allow workers to handle two wire harnesses at the same time, taking into account that one wire harness cannot be completed in a single step as it requires the following steps:

- Place the first wires of the harness on the panel

- Perform two spot tapings

- Place the second wires of the harness

- Perform the remaining five spot tapings

This constraint limited the design of a solution in which the cell is able to handle the four step process described and to maintain the collaborative aspect of the proposed method. The sequence diagram in Fig. 6 reflects the flow of the process for each actor involved in the task.

The finished hardware parts can be visualised in Fig.7 and Fig.8

The back side of the robotic cell prototype contains all the required electronics, including the raspberry pi controller [34], a networking switch, the linear axis PLC and the controllers for both collaborative robots. 




Figure 6: Sequence diagram of the proposed method.

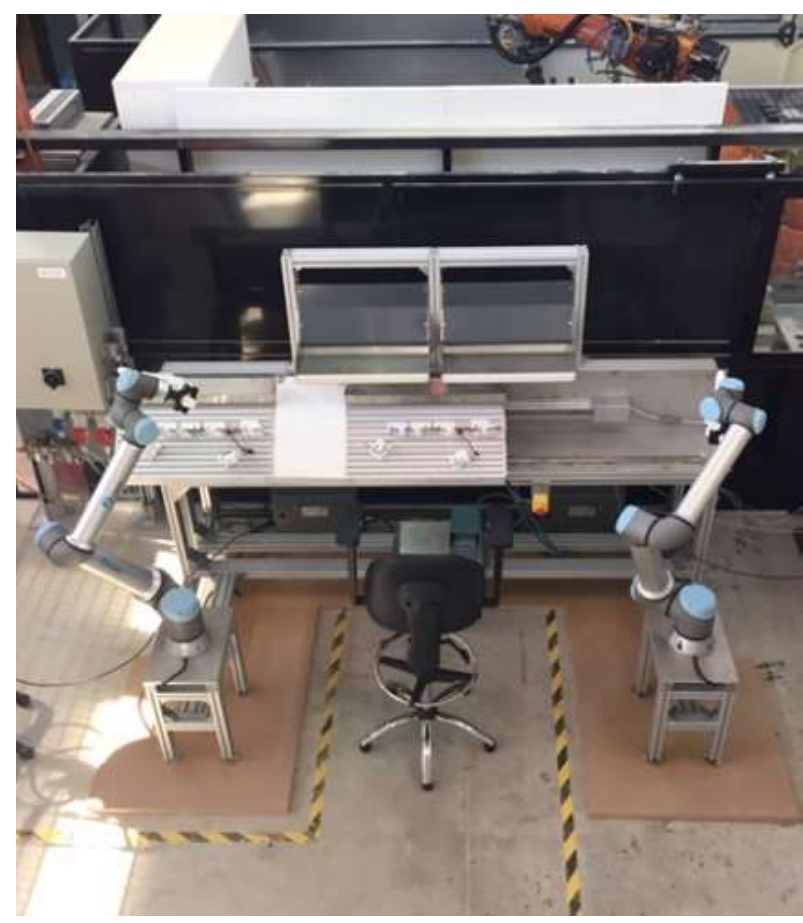

Figure 7: Finished prototype from aerial view.

A simulation has been performed to verify that the design is feasible with the selected materials and components before acquiring them, to avoid unnecessary costs. This simulation was

145 also required to check whether there will be any safety problem 165 [35] with collisions of the robots with other cell elements or with the operator. Fig.9 shows the simulation performed complying with the distance between both in the proposed robotic cell and the reach of each cobot. It shows that the place where

${ }_{150}$ the operator is located while the cell is working in the task is ${ }_{170}$ right in the middle of the reach of both cobots. To avoid hurting workers and damaging the robotic cell itself, two measures



Figure 8: Finished prototype from side view.



Figure 9: Simulation of workspace for both cobots

have been taken into account. The first one is to limit the working space of the tool for each robot to the panel with a minimal offset on the $\mathrm{Z}$ direction to avoid movement between spot points. This measure partially protects workers as it takes into account only the taping spot tool, but not other moving parts of the robot such as shoulder or elbow. The second measure is to put virtual fences on [36] to avoid any moving part of the cobot to go beyond a virtual plane in space. Figure. 10 shows a virtual barrier used on one of the two cobots of the proposed method to enhance safety.

\subsection{Additive manufacturing}

In this project an additive manufacturing has been used to create three-dimensional objects by adding layers of fused extruded material with a technology called Fused Deposition Modeling (FDM) [37]. This additive manufacturing technique allowed to create functional or aesthetic prototypes of parts inside the robotic cell [38]. This was a key factor for the experiments, as it was an iterative process where design changed multiple times after some experimentation of the produced parts, which could have led to expensive machining costs for each change. 




Figure 10: Virtual barrier to increase safety.

The first 3D printed object built has been the spot taping pistols. To start the experiments, two of them were printed with the same weight of $980 \mathrm{gr}$, same proportions and shape as the original ones. It allowed to experiment with them having almost no economic cost and without damaging or breaking the expensive functional taping pistols. The next part to be built was the support that holds the spot taping pistol from the last axis of the collaborative robot. After the design of the support becomes final, it can be machined into a solid metal part, which is unnecessary as great forces are not to be withstand during the spot taping process. The plastic spots used to support the wires while positioning and spot taping follow the Poka Yoke guidelines [39] to ensure users perform the tapings in the only correct place. They were redesigned to reduce its size while maintaining their functionality. Lights inside the spots used in the man- ${ }^{205}$ ual process to visually tell workers the order and next spot to tap were removed as they are no lo longer necessary, since the spot taping process is now being performed by cobots. The latest $3 \mathrm{D}$ printed parts were focused on safety, to avoid human operators to put their hands or fingers between the two panels ${ }^{210}$ and in the rail below them. Support spots and both safety parts can be seen in Fig. 11.



Figure 11: 3DP used for support spots and safety parts.

\begin{tabular}{cccccc}
\hline Method & $T_{\text {pos } 1}$ & $T_{\text {tap } 1}$ & $T_{\text {pos } 2}$ & $T_{\text {tap } 2}$ & $T_{\text {axis }}$ \\
\hline Manual & $14 \mathrm{~s}$ & $4 \mathrm{~s}$ & $9 \mathrm{~s}$ & $11 \mathrm{~s}$ & - \\
Proposed & $14 \mathrm{~s}$ & $2 \mathrm{~s}$ & $9 \mathrm{~s}$ & $7 \mathrm{~s}$ & $2.5 \mathrm{~s}$ \\
\hline
\end{tabular}

Table 1: Measured averaged values for manual and proposed subprocesses.

\section{Cycle time}

To measure the improvement to the cycle time of the wire harnesses spot taping process, the equation Eq3 has been obtained.

$$
\begin{aligned}
T_{R}=\sum_{i=1}^{n} 2 T_{\text {axis }}+ & \frac{1}{2}\left(\max \left\{T_{\text {pos } 1}, T_{\text {tap } 1}\right\}+\max \left\{T_{\text {pos } 1}, T_{\text {tap } 2}\right\}\right. \\
& \left.+\max \left\{T_{\text {pos } 2}, T_{\text {tap } 1}\right\}+\max \left\{T_{\text {pos } 2}, T_{\text {tap } 2}\right\}\right)
\end{aligned}
$$

Being $T_{R}$ the total time required by the proposed solution to finish $n$ wire harnesses, $T_{\text {axis }}$ the time required by the linear axis to move panels from one side to another, $T_{p o s 1}, T_{p o s}$ the time needed by human operators to handle and position the first and second set of wires on the harness and $T_{\text {tap } 1}, T_{\text {tap } 2}$ the time required by the cobots to perform the first and second part of the spot tapings.

The manual process takes an average of 38 seconds to complete a full wire harness. This can be split in multiple subparts to create the equation Eq.4 used to measure the cycle time $T_{O}$ of the manual approach.

$$
T_{O}=\sum_{i=1}^{n} T_{p o s 1}+T_{t a p 1}+T_{p o s 2}+T_{t a p 2}
$$

In this equation, both $T_{\text {tap } 1}$ and $T_{\text {tap } 2}$ refer to the time needed by the human operator to grab the taping pistol and perform the spot tapings for the first and second step of the manual process. Real time values were measured from the manual process to obtain each variable of the equation; those values can be seen in Table.1.

Note that values for $T_{p o s 1}$ and $T_{p o s 2}$ are equal for both manual and proposed method, as they are performed by human operators even in the proposed method, where only the spot taping part of the process is automated. With these values a graph has been generated in Fig. 12 to showcase the comparison between cycle times on both manual process and the proposed method up to a full 8 hour shift. The proposed method reduces the cycle time from 38 to 28 seconds with a $35.8 \%$ increased production and a linear trend. Experiments have been carried out to measure and compare the expected results with the real world scenario of the prototype, leading to the same values. A full four wire harnesses batch were created multiple times with the automated prototype resulting in an average time of 1 minute and 52 seconds which is 28 seconds for a single wire harness. For comparison, the manual process is able to create a maximum of 757 wire harnesses in a single shift while our proposed method is able to build 1028, 271 more harnesses per shift.

\section{Ergonomics}

Tasks in which human workers are present need to take into account their health. To avoid or minimise mid and long term 


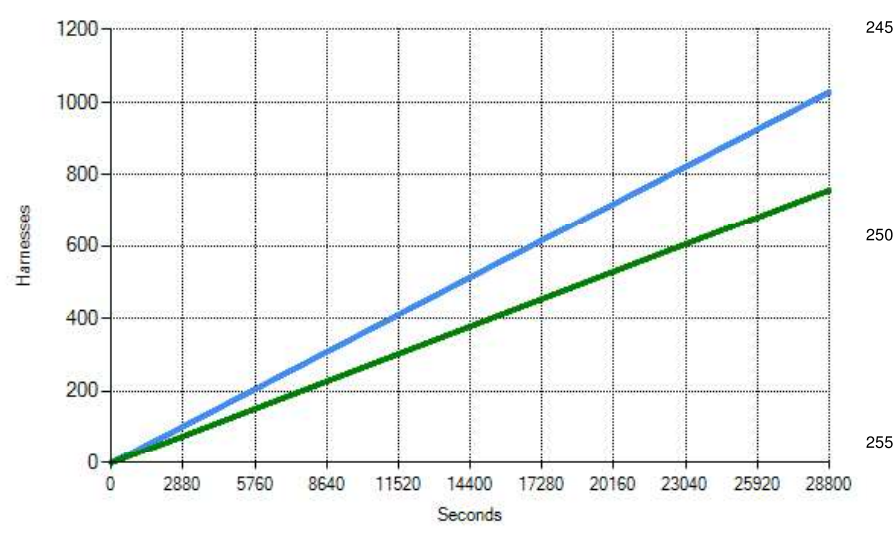

- Proposed - Manual

Figure 12: Cycle time comparison between manual process and proposed solu- ${ }_{260}$ tion.

\begin{tabular}{|c|l|}
\hline Score & Level of MSD Risk \\
\hline $1-2$ & Negligible risk, no action required \\
\hline $3-4$ & Low risk, change may be needed \\
\hline $5-6$ & Medium risk, further investigation, change soon \\
\hline $6+$ & Very high rish, implement change now \\
\hline
\end{tabular}

Table 2: Level of risk depending on RULA score.

health problems of the current process, ergonomics has been studied and improved. The RULA method [40] is a popular ergonomic assessment tool has been used to measure ergonomics objectively both in the actual manual process of the task and in the proposed solution. This indicator will be used to detect if there are ergonomic problems in the actual manual process that need to be fixed and will also allow to compare the actual process with the improved one in the proposed solution. The score in RULA is obtained by measuring location and rotations of different body parts required to perform the desired task [41].

After the RULA score is obtained, it should be compared with the musculoskeletal disorder risk table of the RULA method shown in Fig. 2 to obtain the ergonomic quality of the process and whether actions should be taken to improve it.

The manual process can be seen in Fig.13 To obtain the RULA score of the manual process a set of videos of the current



Figure 13: Manual process of the spot taping task.
245 manual process recorded from different angles have been studied to determine the positions and rotations of different body parts required to perform the spot taping process from the positioning of the cables to the spot taping itself. The obtained score is 7, which, according to the MSD risk table, poses a very high risk and, thus, changes in the ergonomics of the process should be implemented as soon as possible.

To improve the score, a set of changes have been implemented in the proposed method. The most impactful change has been to completely remove the spot taping process from the task, as it is now being performed by a collaborative robot. This reduces the movements required by the human worker to perform the task and obtain a finished wire harness, since now the operator only needs to handle the positioning of wires. The following improvement has been to allow workers to sit while working, but with the correct height to allow a correct positioning of their back. The panels where cables are handled are placed with a proper inclination to minimise the angles required by wrists to handle wires. A pedal is located in the bottom part of the proposed collaborative cell to allow workers to notify that they have finished their current part of the task and allow panels to move to and from cobots without requiring workers to move their hands out of the working panels. With all these design changes to the hardware of the proposed robotic cell, the RULA score has been lowered down to 3. Fig.14 shows a worker handling the proposed robotic cell with the ergonomic improvements included. According to the MSD risk table, the risk is now low and further changes may be needed. The score and the ergonomics itself of the current process have been significantly improved and the risk of health problems minimised but there is still room for improvement for future work.



Figure 14: Worker handling proposed robotic cell with improved ergonomics. 


\section{Scalability}

The minimal proposed setup uses two cobots and a single worker to produce two wire harnesses at the same time. However, this robotic cell has been designed with scalability in mind, allowing to create more complex setups with slight design modifications. The first proposed modification shown in Fig.15 involve mirroring the robotic cell and using the same two cobots, but with two workers to achieve processing four wire ${ }^{300}$ harnesses at the same time. This approach takes advantage of cobot downtimes that occur while the operator is preparing the wire harness of its side to work with the mirror panel located behind the robot, improving the productivity while maintaining the cycle time and without adding more collaborative robots to the design.



Figure 15: Scalable option to produce 4 wire harnesses at the same time

A more extreme version to show the scalability potential of the current proposal is shown in Fig.16. This setup uses four ${ }_{32}$ cobots and four workers to achieve processing sixteen wire harnesses at the same time, maintaining the cycle time but multiplying by eight the production.



Figure 16: Scalable option to produce 16 wire harnesses at the same time
The current design also allows to create further setups that meet the needs of companies implementing the proposed solution.

\section{Conclusions}

Handling of ductile materials is a difficult task to perform with robotics and is one of the keys to automate more manufacturing processes in sectors that use them such as automotive, white goods, textile, shoe-making, etcetera. The automotive sector uses wire harnesses and need to perform tasks with them, which are performed by hand. The proposed method allows human operators to collaborate with robotic arms so their task is enhanced, allowing them to focus only on the handling and positioning part of the job, while the collaborative robots perform the repetitive spot taping process. This enhances the precision of the taping, allows the operator to work with two wire harnesses at the same time increasing revenue and improves the ergonomics of the worker. Additive manufacturing has been another key factor to achieve a functional prototype in time and to perform different experiment and design changes without expending much money. Future works will be focused on the scalability of the proposed solution by experimenting with different setups consisting on more cobots and workers, taking advantage of the working time of cobots to enhance the actual cycle time.

\section{Acknowledgements}

The authors disclosed receipt of the following financial support for the research of this article: This work was supported by ESMERA programme - European SMEs Robotic Applications started in January 2018 and coordinated by the Laboratory for Manufacturing Systems and Automation at the University of Patras. This project has received funding from the European Union's Horizon 2020 research and innovation programme under grant agreement No 780265 .

\section{References}

[1] M. Bell, Flexible object manipulation, Tech. rep. (2010). doi:10.1109/ ROBOT . 2000.844881.

[2] J. E. Hopcroft, J. K. Kearney, D. B. Krafft, Case study of flexible object manipulation, International Journal of Robotics Researchdoi : 10.1177/ 027836499101000105 .

[3] J. Trommnau, J. Kühnle, J. Siegert, R. Inderka, T. Bauernhansl, Overview of the state of the art in the production process of automotive wire harnesses, current research and future trends, in: Procedia CIRP, 2019. doi: 10.1016/j.procir.2019.03.067.

[4] M. Kobayashi, Y. Hirano, M. Higashi, Optimization of Assembly Processes of an Automobile Wire Harness, Computer-Aided Design and Applicationsdoi : 10.1080/16864360.2014.863500.

[5] C. Larsen, J. Sindholt, M. P. Bendsoe, Optimization of compliant mechanisms using genetic algoritms, Ph.D. thesis (2003).

[6] W. P. Pemarathne, T. G. Fernando, Wire and cable routings and harness designing systems with AI, a review, in: 2016 IEEE International Conference on Information and Automation for Sustainability: Interoperable Sustainable Smart Systems for Next Generation, ICIAfS 2016, 2016. doi : 10.1109/ICIAFS. 2016.7946575. 
[7] K. M. Koo, X. Jiang, K. Kikuchi, A. Konno, M. Uchiyama, Development of a robot car wiring system, in: IEEE/ASME International Conference 420 on Advanced Intelligent Mechatronics, AIM, 2008. doi:10.1109/AIM. 2008.4601774 .

[8] K. Oba, Wiring harnesses for Next Generation Automobiles, Fukikura Technical Review.

[9] X. Jiang, Y. Nagaoka, K. Ishii, S. Abiko, T. Tsujita, M. Uchiyama, Robo-425 tized recognition of a wire harness utilizing tracing operation, Robotics and Computer-Integrated Manufacturingdoi:10.1016/j.rcim. 2014. 12.002.

[10] M. Rice, H. H. Tay, J. Ng, C. Lim, S. K. Selvaraj, E. Wu, Comparing three task guidance interfaces for wire harness assembly, in: Conference 430 on Human Factors in Computing Systems - Proceedings, 2016. doi : 10.1145/2851581.2892347.

[11] A. Pradhan, Current Trends in Automotive Wire Harness Design, in: International Conference on Mechanical, Production and Automobile Engineering, 2011. doi:10.1109/MACE.2011.5988240Publication. 435

[12] A. Kumar, G. Chaudhary, M. Kalra, B. K. Jha, Optimization of cycle time for wire harness assembly - Line balancing and Kaizen approach, International Journal of Innovative Research in Science, Engineering and Technologydoi:10.15680/ijirset .2014.0308036.

[13] N. Öztürk, M. N. Esin, Investigation of musculoskeletal symptoms and er-440 gonomic risk factors among female sewing machine operators in Turkey, International Journal of Industrial Ergonomicsdoi : 10.1016/j .ergon . 2011.07.001.

[14] P. Drinkaus, R. Sesek, D. Bloswick, T. Bernard, B. Walton, B. Joseph, G. Reeve, J. H. Counts, Comparison of ergonomic risk assessment outputs 445 from Rapid Upper Limb Assessment and the Strain Index for tasks in automotive assembly plants, Work.

[15] Y. K. Kong, S. yong Lee, K. S. Lee, D. M. Kim, Comparisons of ergonomic evaluation tools (ALLA, RULA, REBA and OWAS) for farm work, International Journal of Occupational Safety and Ergonomicsdoi : 450 $10.1080 / 10803548.2017 .1306960$.

[16] J. R. T. Domingo, M. T. S. Pano, D. A. G. Ecat, N. A. D. Sanchez, B. P. Custodio, Risk Assessment on Filipino Construction Workers, Procedia Manufacturingdoi:10.1016/j.promfg.2015.07.226.

[17] M. Massaccesi, A. Pagnotta, A. Soccetti, M. Masali, C. Masiero,455 F. Greco, Investigation of work-related disorders in truck drivers using RULA method, Applied Ergonomicsdoi:10.1016/S0003-6870(03) 00052-8.

[18] B. M. Deros, D. D. I. Daruis, I. M. Basir, A Study on Ergonomic Awareness among Workers Performing Manual Material Handling Activities,460 Procedia - Social and Behavioral Sciencesdoi:10.1016/j.sbspro. 2015.06.238

[19] S. Sahu, M. Sett, Ergonomic evaluation of tasks performed by female workers in the unorganized sectors of the manual brick manufacturing units in India, Ergonomics SAdoi:10.4314/esa.v22i1.

[20] P. Mukhopadhyay, S. Srivastava, Ergonomic design issues in some craft sectors of Jaipur, Design Journaldoi:10.2752/ $146069210 \times 12580336766446$.

[21] Esben H. Østergaard, The role of cobots in industry 4.0 (2017).

[22] A. Rojko, Industry 4.0 concept: Background and overview, International Journal of Interactive Mobile Technologiesdoi : 10.3991/i jim.v11i5. 7072.

[23] A. Cherubini, R. Passama, A. Crosnier, A. Lasnier, P. Fraisse, Collaborative manufacturing with physical human-robot interaction, Robotics and Computer-Integrated Manufacturingdoi:10.1016/j.rcim. 2015. 12.007.

[24] M. Dudek-Burlikowska, D. Szewieczek, The Poka-Yoke method as an improving quality tool of operations in the process, Journal of Achievements in Materials and Manufacturing Engineering.

[25] R. Szabo, A. Gontean, Industrial robotic automation with Raspberry PI using image processing, in: International Conference on Applied Electronics, 2016. doi:10.1109/AE. 2016.7577287

[26] E. Kadiyala, S. Meda, R. Basani, S. Muthulakshmi, Global industrial process monitoring through IoT using Raspberry pi, in: 2017 International Conference On Nextgen Electronic Technologies: Silicon to Software, ICNETS2 2017, 2017. doi: 10.1109/ICNETS2 . 2017.8067944.

[27] H. K., D. D., Industrial Automation using IoT with Raspberry Pi, International Journal of Computer Applications 168 (1) (2017) 44-48. doi:10.5120/ijca2017914277.
URL http://www.ijcaonline.org/archives/volume168/ number1/merchant-2017-ijca-914277.pdf

[28] T. Birtchnell, J. Urry, A Brief History of 3D Printing, in: A New Industrial Future?, 2018. doi:10.4324/9781315776798-2.

[29] A. Azanha, A. R. T. T. Argoud, J. B. de Camargo Junior, P. D. Antoniolli, Agile project management with Scrum, International Journal of Managing Projects in Businessdoi:10.1108/i jmpb-06-2016-0054.

[30] V. Román-Ibáñez, A. Jimeno-Morenilla, F. Pujol-López, F. Salas-Pérez, Online simulation as a collision prevention layer in automated shoe sole adhesive spraying, International Journal of Advanced Manufacturing Technologydoi:10.1007/s00170-017-1257-2.

[31] P. Jorgensen, Finite State Machines, in: Modeling Software Behavior, 2009. doi:10.1201/b15296-7.

[32] S. El Zaatari, M. Marei, W. Li, Z. Usman, Cobot programming for collaborative industrial tasks: An overview (2019). doi:10.1016/j.robot. 2019.03 .003$.

[33] D. Shreiner, G. Sellers, J. M. Kessenich, B. Licea-Kane, K. O. A. R. B. W. Group, OpenGL Programming Guide: The Official Guide to Learning OpenGL, Version 4.3, Graphics programming, Addison-Wesley, 2013. URL https: //books.google.es/books?id=gnxUewAACAAJ

[34] V. Ramya, G. T. Rajan, Raspberry Pi Based Energy Efficient Industrial Automation System, International Journal of Innovative Research in Computer Science and Engineering.

[35] F. Vicentini, Terminology in safety of collaborative robotics (2020). doi : 10.1016/j.rcim.2019.101921

[36] C. Umstatter, The evolution of virtual fences: A review (2011). doi : 10.1016/j.compag.2010.10.005.

[37] T. D. Ngo, A. Kashani, G. Imbalzano, K. T. Nguyen, D. Hui, Additive manufacturing (3D printing): A review of materials, methods, applications and challenges (2018). doi:10.1016/j.compositesb. 2018 . 02.012 .

[38] I. Gibson, D. Rosen, B. Stucker, Additive manufacturing technologies: 3D printing, rapid prototyping, and direct digital manufacturing, second edition, 2015. doi:10.1007/978-1-4939-2113-3.

[39] P. S. Patil, S. P. Parit, Y. Burali, "Poka Yoke: The Revolutionary Idea In Total Productive Management”, Research Inventy: International Journal Of Engineering And Science Issn Www.Researchinventy.Com.

[40] L. McAtamney, E. Nigel Corlett, RULA: a survey method for the investigation of work-related upper limb disorders, Applied Ergonomicsdoi : 10.1016/0003-6870(93) 90080-S.

[41] M. Middlesworth, A Step-by-Step Guide to the RULA Assessment Tool, Ergonomics Plus. 